\title{
CLASSIFICATION OF BIOTIC INTERACTIONS IN PALEOECOLOGY: AN EVALUATION OF THE EXISTING PARADIGM AND A PROPOSED ENHANCEMENT
}

\author{
GIBSON, Michael A., Dept. of Geology, Geography, Physics, The University of Tennessee at \\ Martin, Martin, TN 38238, U.S.A., mgibson@utm.edu
}

Mutualism, as well as other biotic interactions (commensalism, parasitism, ammensalism, etc.) are behavioral adaptations that play a fundamental role in the ecological and evolutionary success of all groups of organisms. Nearly all organisms enter into one or more types of these relationships at some time during their life history. Studies of the different types of biotic interactions from modern ecosystems demonstrate that the factors associated with biotic interaction ecology are numerous and extremely variable in nature (e.g., timing and duration of association, obligate versus facultative nature, degree of integration of life histories, numbers of participants, side benefits or costs of the relationships, nature of the response, etc.), and yet, ecologists recognize that these interactions often serve as primary "organizing principles" or part of the "rules for assembly" for the communities that the organisms are members. How then do we catagorize, describe, and evaluate them? The nature of the association must be "categorized" first. While there have been numerous philosophies and methodologies to establishing the nature of biotic interactions, the most often used scheme is the cost-benefit analysis approach (Odum Scheme). While this approach appears quantitatively grounded, it is simplistic and inherently qualitative in foundation, and is therefore subject to considerable variation in utilization and confidence as applied to living organisms, for which the system was designed to study. Most often the cost/benefit is only accurately determined by direct observation during the time of interaction because (1) the cost/benefit is reflected using soft-part anatomy or (2) the relationship is visible only as a transient behavior without any permanent structural response from each participant.

Herein lies the problem for paleontologists. Our ability to establish the nature of biotic interactions with confidence lies in the preservability of the relationship (skeletal responses being the most often cited, statistical inference used less frequently). The uncertainty of establishing the nature and cost/benefit of the relationship, ability to identify the ancillary factors that control the relationship, and taphonomic problems inherent to the study of the fossil record have hindered our ability to elucidate evolutionary trends regarding biotic interactions, other than perhaps for predation, or identify the basic "underlying principles" concerning biotic interactions that the through-time perspective of paleontology should be able to give. Rather than blindly apply the system developed for living organisms by neontologists, paleontology needs a system that (1) accurately reflects our assessment of the nature of the relationship, (2) accurately conveys our confidence in our interpretation (e.g., the Boucot Reliability Classes), (3) is user-friendly, and (4) is compatible with the system employed in neontology. After a review of the historical development of the problems associated with the terminology and classifications devised for biotic interaction ecology/paleoecology, I present a classification scheme for use in paleontology that incorporates the established neontology-based cost/benefit approach, Boucot's Reliability Classes, and additional levels that should allow paleontologists to better identify interactions such as mutualism from the fossil record, communicate biotic interaction information, and provide a common ground upon which biotic interaction paleoecology can begin to define the foundation principles of the science. 